\title{
Plasma vitamin $D$ is associated with fasting insulin and homeostatic model assessment of insulin resistance in young adult males, but not females, of the Jerusalem Perinatal Study
}

\author{
Amy Moore ${ }^{1}$, Hagit Hochner ${ }^{2}$, Colleen M Sitlani ${ }^{3}$, Michelle A Williams $^{4}$, \\ Andrew N Hoofnagle ${ }^{5,6}$, lan $\mathrm{H}$ de Boer ${ }^{1,6}$, Bryan Kestenbaum 1,6, David S Siscovick 1,3,6, \\ Yechiel Friedlander ${ }^{2}$ and Daniel A Enquobahrie ${ }^{1,3,7, *}$ \\ 'Department of Epidemiology, University of Washington, Cardiovascular Health Research Unit, 1730 Minor \\ Avenue, Suite \# 1360, Seattle, WA 98101, USA: ${ }^{2}$ Braun School of Public Health, Hebrew University Hadassah \\ Medical Center, Jerusalem, Jerusalem, Israel: ${ }^{3}$ Cardiovascular Health Research Unit, University of Washington, \\ Seattle, WA, USA: ${ }^{4}$ Department of Epidemiology, Harvard School of Public Health, Harvard University, Boston, MA, \\ USA: ${ }^{5}$ Department of Laboratory Medicine, University of Washington, Seattle, WA, USA: ${ }^{6}$ Department of Medicine, \\ University of Washington, Seattle, WA, USA: ${ }^{7}$ Center for Perinatal Studies, Swedish Medical Center, Seattle, WA, USA
}

Submitted 18 November 2013: Final revision received 25 April 2014: Accepted 18 June 2014: First published online 13 August 2014

\begin{abstract}
Objective: To examine cross-sectional relationships between plasma vitamin D and cardiometabolic risk factors in young adults.

Design: Data were collected from interviews, physical examinations and biomarker measurements. Total plasma 25-hydroxyvitamin D (25(OH)D) was measured using LC-tandem MS. Associations between 25(OH)D and cardiometabolic risk factors were modelled using weighted linear regression with robust estimates of standard errors.

Setting: Individuals born in Jerusalem during 1974-1976.

Subjects: Participants of the Jerusalem Perinatal Study ( $n$ 1204) interviewed and examined at age 32 years. Participants were oversampled for low and high birth weight and for maternal pre-pregnancy obesity.

Results: Mean total 25(OH)D concentration among participants was 21.7 (SD 8.9) $\mathrm{ng} / \mathrm{ml}$. Among males, 25(OH)D was associated with homeostatic model assessment of insulin resistance (natural log-transformed, $\beta=-0 \cdot 011, P=0 \cdot 004$ ) after adjustment for BMI. However, these associations were not present among females $(P$ for sex interaction $=0 \cdot 005)$.

Conclusions: We found evidence for inverse associations of $25(\mathrm{OH}) \mathrm{D}$ with markers of insulin resistance among males, but not females, in a healthy, young adult Caucasian population. Prospective studies and studies conducted on other populations investigating sex-specific effects of vitamin D on cardiometabolic risk factors are warranted.
\end{abstract}

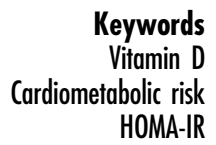

HOMA-IR
Vitamin D receptors are ubiquitous in the body ${ }^{(1)}$, indicating widespread effects of vitamin $\mathrm{D}$ in health and disease. Adverse effects of vitamin D deficiency, defined by low circulating concentrations of 25-hydroxyvitamin D $(25(\mathrm{OH}) \mathrm{D})$, on bone health have long been known ${ }^{(2)}$. More recently, potential associations of vitamin $\mathrm{D}$ with other chronic diseases, including cancer, CVD, autoimmune disease and type 2 diabetes (T2D), have been demonstrated $^{(3)}$. For instance, prospective cohort studies have found associations between baseline low circulating $25(\mathrm{OH}) \mathrm{D}$ concentrations and incident myocardial infarction, stroke and all-cause mortality ${ }^{(4-6)}$. However, findings from randomized clinical trials of vitamin D supplementation to reduce hypertension and cardiovascular mortality were inconsistent ${ }^{(7)}$, suggesting more complex relationships between vitamin $\mathrm{D}$ and cardiovascular risk development.

A clear understanding of relationships between vitamin $\mathrm{D}$ and known cardiometabolic risk factors (CMR), such as fasting blood glucose and related parameters, blood pressure (BP) and lipid levels, as well as the role of potential confounders or mediators, such as obesity, is lacking, particularly among young adults free from overt disease. Further, sex-specific differences have not been fully explored, although it is recognized that men tend to 
have higher $25(\mathrm{OH}) \mathrm{D}$ levels than women ${ }^{(8)}$ while having an earlier age of onset of CMR development ${ }^{(9)}$.

We examined cross-sectional relationships between circulating $25(\mathrm{OH}) \mathrm{D}$ and CMR among young adult participants of a longitudinal birth cohort study, the Jerusalem Perinatal Study (JPS). We also evaluated whether these relationships differ by sex or are confounded by current BMI.

\section{Methods}

\section{Overall setting and study population}

The JPS was conducted among women who gave birth as residents of Jerusalem between 1974 and 1976 and who were interviewed within $2 \mathrm{~d}$ postpartum ( $n$ 17 003). The JPS Family Follow-Up study (JPS-1), conducted between 2007 and 2009, was a study designed to determine maternal and offspring genetic risk factors that account for associations between maternal obesity and adulthood CMR among the offspring. The JPS-1 study population consisted of mother-offspring dyads identified and recruited using a stratified sampling of term, singleton live births from the original JPS cohort. Participants were oversampled for low $(\leq 2500 \mathrm{~g})$ and high $(\geq 4000 \mathrm{~g})$ birth weight and for maternal obesity (pre-pregnancy BMI $\geq 27 \mathrm{~kg} / \mathrm{m}^{2}$ ). Mother-offspring dyads with offspring who had congenital disorders at birth were excluded.

The current study was conducted among offspring JPS-1 participants, 32 (SD 1.3) years old on average. Individuals who self-reported taking medication to lower BP ( $n$ 13), lipids ( $n$ 14) or to treat diabetes ( $n$ 5) were excluded from the analyses. Individuals with a fasting time before blood draw of less than $8 \mathrm{~h}$ ( $n$ 97), fasting glucose outside the range $60-126 \mathrm{mg} / \mathrm{dl}$ ( $n$ 135) or TAG higher than $600 \mathrm{mg} / \mathrm{dl}$ ( $n$ 58) were excluded. The final analytic study population consisted of 1204 offspring (609 males and 595 females). Study procedures were approved by the Institutional Review Boards of the University of Washington, in Seattle, and the Hebrew University Hadassah Medical Center in Jerusalem. All participants provided informed consent.

\section{Data collection}

Data collection procedures have been published previously $^{(10)}$. Briefly, data on anthropomorphic measurements, lifestyle and sociodemographic characteristics were collected during offspring interviews conducted between 2007 and 2009. BMI was calculated as weight $(\mathrm{kg})$ divided by squared height $\left(\mathrm{m}^{2}\right)$. BP was measured as the average of three consecutive measurements performed after sitting for 5 min (Omron M7 automated sphygmomanometer). Fasting blood samples were taken using standard procedures. Plasma glucose, HDL-cholesterol (HDL-C) and TAG were measured on the VITROS 5,1FS Chemistry System (Ortho Clinical Diagnostics, Raritan, NJ, USA). Plasma insulin levels were determined using RIA with the Human Insulin-Specific RIA Kit (Millipore, Billerica, MA, USA).
Plasma vitamin D (25(OH)D) was measured using LCtandem MS at the Nutrition and Obesity Research Center, University of Washington (Seattle, WA, USA). The sum of $25(\mathrm{OH}) \mathrm{D}_{2}$ and $25(\mathrm{OH}) \mathrm{D}_{3}$ was used in analyses to represent total plasma vitamin D levels.

\section{Statistical analyses}

We examined participant characteristics in the entire study cohort and stratified by sex. Means and standard deviations were calculated for continuous variables; numbers and percentages for categorical variables. We used weighted multiple linear regression models to examine associations between offspring 25(OH)D and CMR (BMI, waist circumference, waist:hip ratio, fasting glucose, fasting insulin, homeostatic model assessment of insulin resistance (HOMA-IR), lipids and BP). Ninety-five per cent confidence intervals and $P$ values were calculated using robust standard errors. BMI, TAG, LDL-cholesterol (LDL-C) and HDL-C were natural log-transformed to improve model fit. We first examined the relationships among the entire study population with sex as a covariate and an adjustment for multiple testing (critical $P$ value $\alpha=0.0045$ ). Then, we used an interaction term between sex and $25(\mathrm{OH}) \mathrm{D}$ to evaluate statistical interaction (at the $P=0.05$ level) between vitamin $\mathrm{D}$ and sex on CMR.

We fit two models to evaluate the associations. Model 1 included season of blood draw (December-February, March-May, June-August, September-November) and additional covariates for smoking status (current, former and never), alcohol abstinence (yes/no), intense physical activity participation (yes/no), employment status (yes/ no), religiosity (ultra-orthodox, religious and traditionalist/ secular/other), ethnic origin (Israel, Morocco, Other Africa, Iraq, Iran, Kurdistan, Yemen, Other Asia and Ashkenazi) and parental history of relevant disease (see below) as confounders and/or to increase precision. The models for fasting blood glucose, insulin and HOMA-IR were adjusted for parental history of diabetes. The models for LDL-C, HDL-C and TAG were adjusted for parental history of heart disease, stroke or elevated cholesterol. The models for systolic and diastolic BP were adjusted for parental history of high BP and stroke. In Model 2, we adjusted for Model 1 variables and BMI, except for the model evaluating BMI as an outcome. The aim of Model 2 was to assess the extent to which BMI mediates or confounds observed associations.

Analyses were performed using the statistical software package Stata v11.0.

\section{Results}

Selected characteristics of the study participants are shown in Table 1. Mean total 25(OH)D level was 21.7, 22.9 and $20.5 \mathrm{mmol} / \mathrm{l}$ among all participants, males and females, respectively. Overall, $44 \%$ of participants, $36 \%$ of males 
Table 1 Characteristics of the study participants by sex and quartile of plasma vitamin D; offspring ( $n$ 1204) in the Jerusalem Perinatal Study (JPS; 1974-1976), interviewed and examined at age 32 years in the JPS Family Follow-Up study (JPS-1; 2007-2009)

\begin{tabular}{|c|c|c|c|c|c|c|c|c|c|c|c|c|c|c|}
\hline & & & & & & & \multicolumn{8}{|c|}{ Quartile of plasma vitamin D (ng/ml) } \\
\hline & \multicolumn{2}{|c|}{ Total† } & \multicolumn{2}{|c|}{ Males } & \multicolumn{2}{|c|}{ Females } & \multicolumn{2}{|c|}{$1(<15.4)$} & \multicolumn{2}{|c|}{$2(15 \cdot 4-21 \cdot 4)$} & \multicolumn{2}{|c|}{$3(21.5-27 \cdot 4)$} & \multicolumn{2}{|c|}{$4(>27 \cdot 4)$} \\
\hline & $n$ or Mean & $\%$ or $\mathrm{SD}$ & $n$ or Mean & $\%$ or SD & $n$ or Mean & $\%$ or SD & $n$ or Mean & $\%$ or SD & $n$ or Mean & $\%$ or SD & $n$ or Mean & $\%$ or SD & $n$ or Mean & $\%$ or SD \\
\hline$n$ & 1204 & - & 609 & - & 595 & - & 301 & - & 306 & - & 288 & - & 294 & - \\
\hline Plasma 25(OH)D (ng/ml) & 21.7 & 8.9 & 22.9 & 8.4 & 20.5 & 9.2 & $11 \cdot 2$ & 2.9 & 18.4 & 1.8 & $24 \cdot 3$ & 1.8 & 33.5 & $5 \cdot 8$ \\
\hline Females $(n, \%)$ & 595 & $49 \cdot 4$ & - & - & - & - & 211 & $61 \cdot 3$ & 184 & $52 \cdot 9$ & 138 & $41 \cdot 0$ & 145 & $42 \cdot 2$ \\
\hline Males $(n, \%)$ & 609 & $50 \cdot 6$ & - & - & - & - & 133 & 38.7 & 164 & $47 \cdot 1$ & 199 & $59 \cdot 1$ & 199 & 57.9 \\
\hline BMI $\left(\mathrm{kg} / \mathrm{m}^{2}\right)$ & $26 \cdot 4$ & $5 \cdot 1$ & $26 \cdot 8$ & 8.9 & $26 \cdot 0$ & $5 \cdot 6$ & $27 \cdot 2$ & 5.8 & $27 \cdot 0$ & 5.8 & $26 \cdot 4$ & 4.4 & $25 \cdot 0$ & 3.8 \\
\hline Fasting blood glucose (mg/dl) & $80 \cdot 3$ & $9 \cdot 0$ & $82 \cdot 3$ & 8.9 & $78 \cdot 3$ & 8.6 & $80 \cdot 8$ & $9 \cdot 0$ & 80.4 & $9 \cdot 0$ & $80 \cdot 8$ & $9 \cdot 3$ & 79.4 & 8.8 \\
\hline TAG (mg/dl) & $103 \cdot 3$ & 61.6 & $116 \cdot 0$ & $69 \cdot 2$ & $90 \cdot 2$ & $49 \cdot 6$ & $102 \cdot 0$ & $65 \cdot 0$ & $105 \cdot 6$ & 61.9 & 107.7 & 65.4 & $99 \cdot 2$ & $53 \cdot 8$ \\
\hline LDL-C (mg/dl) & 112.2 & 28.4 & $117 \cdot 2$ & $28 \cdot 3$ & $107 \cdot 2$ & $27 \cdot 6$ & 110.4 & $27 \cdot 8$ & 111.8 & 27.4 & $115 \cdot 2$ & $30 \cdot 6$ & 111.4 & 27.9 \\
\hline $\mathrm{HDL}-\mathrm{C}(\mathrm{mg} / \mathrm{dl})$ & 49.9 & 14.5 & $43 . \overline{5}$ & $11 \cdot 1$ & $56 \cdot \overline{4}$ & $14 \cdot 8$ & 51.2 & $15 \cdot 1$ & 48.8 & 13.9 & 48.8 & 14.4 & 50.4 & $14 \cdot 2$ \\
\hline Systolic BP (mmHg) & $106 \cdot 4$ & $12 \cdot 3$ & $113 \cdot 8$ & $10 \cdot 4$ & 98.8 & $9 \cdot 2$ & $105 \cdot 5$ & 11.9 & $107 \cdot 0$ & $12 \cdot 7$ & 107.8 & 12.5 & $105 \cdot 4$ & $12 \cdot 0$ \\
\hline Diastolic BP (mmHg) & 71.6 & 8.5 & 74.7 & 7.9 & 68.5 & $7 \cdot \overline{8}$ & 71.9 & 8.9 & $72 \cdot 2$ & 8.5 & 72.2 & $8 \cdot 3$ & 70.4 & 8.1 \\
\hline Fasting insulin ( $\mu \mathrm{lU} / \mathrm{ml})$ & $12 \cdot 0$ & $7 \cdot 2$ & $12 \cdot 6$ & $7 \cdot 4$ & 11.5 & 6.9 & $13 \cdot 1$ & $7 \cdot 8$ & 13.5 & 8.8 & 11.4 & $5 \cdot 6$ & $10 \cdot 2$ & $5 \cdot 4$ \\
\hline HOMA-IR & 2.4 & 1.5 & $2 \cdot 6$ & 1.6 & $2 \cdot 2$ & 1.4 & $2 \cdot 6$ & 1.6 & $2 \cdot 7$ & 1.8 & $2 \cdot 3$ & 1.3 & $2 \cdot 0$ & 1.2 \\
\hline Waist circumference $(\mathrm{cm})$ & $86 \cdot 5$ & $13 \cdot 1$ & 91.1 & 11.8 & 81.8 & $12 \cdot 6$ & 88.2 & $14 \cdot 3$ & $87 \cdot 7$ & 14.2 & 86.7 & $11 \cdot 7$ & 83.7 & 11.3 \\
\hline Waist:hip ratio & 0.83 & 0.07 & 0.87 & 0.06 & 0.79 & 0.06 & 0.83 & 0.08 & 0.83 & 0.07 & 0.83 & 0.07 & 0.82 & 0.07 \\
\hline \multicolumn{15}{|l|}{ Smoking status $(n, \%)$} \\
\hline Former & 161 & 13.7 & 90 & $15 \cdot 2$ & 71 & $12 \cdot 1$ & 39 & $13 \cdot 1$ & 37 & $12 \cdot 5$ & 42 & $15 \cdot 1$ & 41 & $14 \cdot 2$ \\
\hline Current & 313 & $26 \cdot 6$ & 210 & 35.5 & 103 & $17 \cdot 6$ & 51 & $17 \cdot 1$ & 66 & $22 \cdot 3$ & 93 & $33 \cdot 3$ & 97 & $33 \cdot 7$ \\
\hline \multicolumn{15}{|l|}{ Abstain from alcohol $(n, \%)$} \\
\hline $\begin{array}{l}\text { Physical activity } \ddagger(n, \%) \\
\text { Any }\end{array}$ & 252 & 000 & 224 & 277 & 120 & ח2 & & \multicolumn{6}{|c|}{ Physical activity $\ddagger(n, \%)$} & \\
\hline \multicolumn{15}{|l|}{ Employment status ( $n, \%)$} \\
\hline Employed & 978 & $84 \cdot 2$ & 521 & $89 \cdot 1$ & 547 & $79 \cdot 3$ & 203 & 69.8 & 245 & $84 \cdot 2$ & 252 & $90 \cdot 0$ & 266 & 93.7 \\
\hline \multicolumn{15}{|l|}{ Religious orthodoxy $(n, \%)$} \\
\hline Traditionalist, secular or other & 737 & $61 \cdot 2$ & 380 & $62 \cdot 4$ & 357 & $60 \cdot 0$ & 101 & 33.6 & 183 & $59 \cdot 8$ & 208 & $72 \cdot 2$ & 233 & $79 \cdot 3$ \\
\hline Religious & 236 & $19 \cdot 6$ & 124 & $20 \cdot 4$ & 112 & $18 \cdot 8$ & 54 & 17.9 & 68 & $22 \cdot 2$ & 58 & $20 \cdot 1$ & 54 & $18 \cdot 4$ \\
\hline Ultra-orthodox & 231 & $19 \cdot 2$ & 105 & $17 \cdot 2$ & 126 & $21 \cdot 2$ & 146 & 48.5 & 55 & $18 \cdot 0$ & 22 & 7.6 & 7 & $2 \cdot 4$ \\
\hline \multicolumn{15}{|l|}{ Blood draw month ( $n, \%)$} \\
\hline December-February & 333 & $27 \cdot 7$ & 164 & $26 \cdot 9$ & 169 & 28.4 & 156 & $51 \cdot 8$ & 91 & 29.7 & 56 & 19.4 & 29 & 9.9 \\
\hline March-May & 198 & $16 \cdot 5$ & 91 & 14.9 & 107 & 18.0 & 58 & $19 \cdot 3$ & 61 & 19.9 & 49 & $17 \cdot 0$ & 26 & 8.8 \\
\hline September-November & 337 & 28.0 & 159 & $26 \cdot 1$ & 178 & 29.9 & 48 & $16 \cdot 0$ & 82 & $26 \cdot 8$ & 96 & 33.3 & 109 & $37 \cdot 1$ \\
\hline
\end{tabular}

25(OH)D, 25-hydroxyvitamin D; LDL-C, LDL-cholesterol; HDL-C, HDL-cholesterol; BP, blood pressure; HOMA-IR, homeostatic model assessment of insulin resistance. tMay not sum to total due to missing data.

$\ddagger$ Defined as exercise that brings about laboured breathing, increased pulse rate and sweating, lasting for at least 20 min 
Table 2 Overall associations between plasma $25(\mathrm{OH}) \mathrm{D}(\mathrm{mmol} / \mathrm{l})$ and cardiometabolic risk factors in young adults; offspring $(n$ 1204) in the Jerusalem Perinatal Study (JPS; 1974-1976), interviewed and examined at age 32 years in the JPS Family Follow-Up study (JPS-1; 2007-2009)

\begin{tabular}{|c|c|c|c|c|c|c|}
\hline & \multicolumn{3}{|c|}{ Model 1} & \multicolumn{3}{|c|}{ Model 2} \\
\hline & Coefficient & SE & $P$ value & Coefficient & SE & $P$ value \\
\hline Glucose (mmol/l) & -0.055 & 0.056 & 0.327 & -0.041 & 0.056 & 0.464 \\
\hline $\operatorname{Ln}(\mathrm{TAG})(\mathrm{mmol} / \mathrm{l})$ & -0.010 & 0.004 & 0.005 & -0.007 & 0.003 & 0.041 \\
\hline Ln(LDL-C) (mmol/l) & -0.001 & 0.002 & 0.488 & 0.000 & 0.002 & 0.836 \\
\hline $\mathrm{Ln}(\mathrm{HDL}-\mathrm{C})(\mathrm{mmol} / \mathrm{l})$ & 0.004 & 0.002 & 0.010 & 0.003 & 0.002 & 0.104 \\
\hline Systolic BP (mmHg) & -0.081 & 0.058 & 0.165 & -0.015 & 0.053 & 0.773 \\
\hline Diastolic BP (mmHg) & -0.108 & 0.046 & 0.020 & -0.061 & 0.043 & 0.157 \\
\hline $\operatorname{Ln}(\mathrm{BMI})\left(\mathrm{kg} / \mathrm{m}^{2}\right)$ & -0.002 & 0.001 & 0.030 & -0.002 & 0.001 & 0.030 \\
\hline Ln(Insulin) (pmol/l) & -0.007 & 0.003 & $0.044^{*}$ & -0.004 & 0.003 & $0.213^{\star}$ \\
\hline Ln(HOMA-IR $)$ & -0.007 & 0.003 & $0.034^{*}$ & -0.004 & 0.003 & $0.174^{*}$ \\
\hline Waist circumference $(\mathrm{cm})$ & -0.132 & 0.069 & 0.056 & -0.003 & 0.036 & 0.929 \\
\hline Waist:hip ratio & 0.000 & 0.000 & 0.196 & 0.000 & 0.000 & 0.700 \\
\hline
\end{tabular}

25(OH)D, 25-hydroxyvitamin D; LDL-C, LDL-cholesterol; HDL-C, HDL-cholesterol; BP, blood pressure; HOMA-IR, homeostatic model assessment of insulin resistance.

Model 1 adjusted for season of blood draw, sex, smoking status, alcohol abstinence, religious orthodoxy, physical activity, employment status, ethnic origin and parental history of disease.

Model 2 adjusted for Model 2 covariates, plus BMI.

${ }^{*} P$ value for $25(\mathrm{OH}) \mathrm{D}$-sex interaction less than 0.05 .

Table 3 Associations between plasma 25(OH)D (mmol/l) and cardiometabolic risk factors among adult females; offspring $(n 595)$ in the Jerusalem Perinatal Study (JPS; 1974-1976), interviewed and examined at age 32 years in the JPS Family Follow-Up study (JPS-1; 2007-2009)

\begin{tabular}{|c|c|c|c|c|c|c|}
\hline & \multicolumn{3}{|c|}{ Model 1} & \multicolumn{3}{|c|}{ Model 2} \\
\hline & Coefficient & $\mathrm{SE}$ & $P$ value & Coefficient & SE & $P$ value \\
\hline Glucose $(\mathrm{mmol} / \mathrm{l})$ & -0.016 & 0.065 & 0.801 & -0.003 & 0.065 & 0.963 \\
\hline Ln(TAG) (mmol/l) & -0.005 & 0.004 & 0.148 & -0.002 & 0.003 & 0.544 \\
\hline Ln(LDL-C) $(\mathrm{mmol} / \mathrm{l})$ & -0.001 & 0.002 & 0.599 & 0.000 & 0.002 & 0.901 \\
\hline Ln(HDL-C) (mmol/li) & 0.003 & 0.002 & 0.193 & 0.001 & 0.002 & 0.606 \\
\hline Systolic BP (mmHg) & -0.126 & 0.063 & 0.045 & -0.055 & 0.060 & 0.362 \\
\hline Diastolic BP (mmHg) & -0.071 & 0.053 & 0.180 & -0.021 & 0.051 & 0.678 \\
\hline $\operatorname{Ln}(\mathrm{BMI})\left(\mathrm{kg} / \mathrm{m}^{2}\right)$ & -0.003 & 0.001 & 0.038 & -0.003 & 0.001 & 0.038 \\
\hline Ln(Insulin) (pmol/l) & 0.000 & 0.004 & 0.947 & 0.003 & 0.004 & 0.379 \\
\hline Ln(HOMA-IR $)$ & -0.001 & 0.004 & 0.886 & 0.003 & 0.004 & 0.431 \\
\hline Waist circumference $(\mathrm{cm})$ & -0.114 & 0.090 & 0.205 & 0.043 & 0.049 & 0.377 \\
\hline Waist:hip ratio & 0.000 & 0.000 & 0.417 & 0.000 & 0.000 & 0.910 \\
\hline
\end{tabular}

25(OH)D, 25-hydroxyvitamin D; LDL-C, LDL-cholesterol; HDL-C, HDL-cholesterol; BP, blood pressure; HOMA-IR, homeostatic model assessment of insulin resistance.

Model 1 adjusted for season of blood draw, smoking status, alcohol abstinance, religious orthodoxy, physical activity, employment status, ethnic origin and parental history of disease.

Model 2 adjusted for Model 1 covariates, plus BMI.

and $52 \%$ of females, had vitamin D deficiency based on the cut-off of $20 \mathrm{ng} / \mathrm{ml}^{(11)}$. Males had higher BMI, fasting blood glucose, TAG, LDL-C, systolic BP, diastolic BP and HOMA-IR compared with females. They also had lower HDL-C levels.

Among all study participants (Table 2), the association between $25(\mathrm{OH}) \mathrm{D}$ with TAG was nearly statistically significant in Model 1 after adjustment for other confounders ( $P>0.0045$ after multiple testing correction). Each $1 \mathrm{mmol} / \mathrm{l}$ increase in $25(\mathrm{OH}) \mathrm{D}$ was associated with a $1 \%$ decrease in TAG levels $(P=0 \cdot 005)$. However, the association was attenuated and became non-significant after adjustment for current BMI ( $P=0.041)$. Among females (Table 3), 25(OH)D was not significantly associated with any outcome of interest. Among males (Table 4), 25(OH)D was inversely associated with insulin and HOMA-IR $(P=0.003$ and 0.002, respectively). Associations of 25(OH)D with HOMA-IR persisted even after adjustment for current BMI ( $P$-value $=0 \cdot 004)$. Significant statistical interactions were observed between 25(OH)D and sex on insulin and HOMA-IR $(P=0.015$ and 0.012 , respectively). We did not observe associations between 25(OH)D and fasting glucose level, LDL-C or BP overall, or among either sex.

\section{Discussion}

In the current study, we found that associations of total plasma $25(\mathrm{OH}) \mathrm{D}$ with fasting insulin concentration and HOMA-IR were present among males but not females. Interactions 
Table 4 Associations between plasma $25(\mathrm{OH}) \mathrm{D}(\mathrm{mmol} / \mathrm{l})$ and cardiometabolic risk factors among adult males; offspring $(n$ 609) in the Jerusalem Perinatal Study (JPS; 1974-1976), interviewed and examined at age 32 years in the JPS Family Follow-Up study (JPS-1; 2007-2009)

\begin{tabular}{|c|c|c|c|c|c|c|}
\hline & \multicolumn{3}{|c|}{ Model 1} & \multicolumn{3}{|c|}{ Model 2} \\
\hline & Coefficient & SE & $P$ value & Coefficient & SE & $P$ value \\
\hline Glucose $(\mathrm{mmol} / \mathrm{l})$ & -0.095 & 0.071 & 0.184 & -0.080 & 0.071 & 0.260 \\
\hline $\operatorname{Ln}(\mathrm{TAG})(\mathrm{mmol} / \mathrm{l})$ & -0.015 & 0.005 & 0.003 & -0.012 & 0.005 & 0.012 \\
\hline Ln(LDL-C) $(\mathrm{mmol} / \mathrm{l})$ & -0.001 & 0.002 & 0.615 & 0.000 & 0.002 & 0.853 \\
\hline Ln(HDL-C) $(\mathrm{mmol} / \mathrm{l})$ & 0.006 & 0.002 & 0.006 & 0.004 & 0.002 & 0.033 \\
\hline Systolic BP (mmHg) & -0.031 & 0.085 & 0.716 & 0.028 & 0.077 & 0.715 \\
\hline Diastolic BP (mmHg) & -0.148 & 0.065 & 0.023 & -0.105 & 0.061 & 0.083 \\
\hline $\operatorname{Ln}(\mathrm{BMI})\left(\mathrm{kg} / \mathrm{m}^{2}\right)$ & -0.002 & 0.001 & 0.195 & -0.002 & 0.001 & 0.195 \\
\hline Ln(Insulin) (pmol/l) & -0.013 & 0.004 & $0.003^{*}$ & -0.010 & 0.004 & 0.006 \\
\hline Ln(HOMA-IR $)$ & -0.014 & 0.004 & $0.002^{*}$ & -0.011 & 0.004 & $0.004^{*}$ \\
\hline Waist circumference $(\mathrm{cm})$ & -0.151 & 0.084 & 0.072 & -0.051 & 0.042 & 0.224 \\
\hline Waist:hip ratio & -0.001 & 0.000 & 0.206 & 0.000 & 0.000 & 0.449 \\
\hline
\end{tabular}

25(OH)D, 25-hydroxyvitamin D; LDL-C, LDL-cholesterol; HDL-C, HDL-cholesterol; BP, blood pressure; HOMA-IR, homeostatic model assessment of insulin resistance.

Model 1 adjusted for season of blood draw, smoking status, alcohol abstinance, religious orthodoxy, physical activity, employment status, ethnic origin and parental history of disease.

Model 2 adjusted for Model 1 covariates, plus BMI.

${ }^{\star} P$ value less than 0.0045 (multiple-testing corrected).

between sex and vitamin D on insulin and HOMA-IR were statistically significant. Among males, the association between fasting insulin concentration and $25(\mathrm{OH}) \mathrm{D}$ was attenuated after adjustment for BMI, such that it no longer met our stringent criteria for statistical significance.

The inverse association between $25(\mathrm{OH}) \mathrm{D}$ and TAG has been widely reported, although many studies report significant associations after adjustment for measures of adiposity, such as BMI ${ }^{(12)}$. In one such study by Kayaniyil et al., the relationship was attenuated with the addition of BMI to the model but remained statistically significant in their slightly older, more obese and sicker population ${ }^{(13)}$. It is therefore possible that the relationship between $25(\mathrm{OH}) \mathrm{D}$ and TAG is stronger and less subject to attenuation by adiposity among individuals with diagnosed metabolic syndrome, potentially explaining the differences between our study and that by Kayaniyil et al.

Evidence for direct effects of vitamin D on TAG levels is still conflicting ${ }^{(12)}$. Only one randomized controlled trial has reported a significant decrease in TAG levels after vitamin D supplementation $^{(14)}$, while four other randomized controlled trials have failed to duplicate these findings ${ }^{(15-18)}$, including one with both a larger sample size and a larger dose of vitamin $\mathrm{D}^{(15)}$. While indirect effects via vitamin D's influence on intestinal Ca absorption are not thought to cause substantial changes in serum $\mathrm{TAG}^{(19)}$, it is plausible that vitamin D reduces serum TAG by suppressing pituitary hormone levels ${ }^{(20)}$ or through its posited effects on glucose metabolism ${ }^{(21)}$, but these hypotheses require further study.

Our analysis of $25(\mathrm{OH}) \mathrm{D}$ and concurrently measured fasting insulin in the JPS offspring population found an inverse association between vitamin D and fasting insulin in males, but not females. To confirm these findings in less constrained models, we also examined models using splines and categories of vitamin D insufficiency and insulin resistance (results not shown), but these models did not add anything to our report. Two other studies have examined these sex-specific associations ${ }^{(22,23)}$ and reported associations in females as well as in males. The study by Delvin et al. examined French-Canadian adolescents who were 9, 13 and 16 years old during the study ${ }^{(23)}$. Due to the age difference, results from that study of adolescents and our study on JPS adults, approximately 32 years old, may not be directly comparable. In their study, Yin et al. excluded individuals who used vitamin D supplements, smoked or engaged in strenuous exercise, besides individuals excluded for other reasons. Furthermore, only $25 \%$ of their study participants were female ${ }^{(22)}$. While we adjusted for most of these factors in our study, study population differences, such as diet or genetics, may account for differences in findings from these studies compared with the current study. Other large, populationbased studies have also found inverse associations between measures of vitamin D and fasting insulin ${ }^{(24-27)}$ in both sexes; however, these studies did not evaluate sexspecific relationships.

We examined a vitamin D-sex interaction due to the literature supporting sex differences in vitamin D levels ${ }^{(8)}$ and cardiometabolic outcomes ${ }^{(9)}$. Despite differences between the results of the current analysis and previous studies $^{(22,23,28)}$, some potential reasons supporting observed associations of $25(\mathrm{OH}) \mathrm{D}$ levels with fasting insulin and HOMA-IR among males, but not females, exist. Among non-diabetics, insulin resistance is higher among males than females ${ }^{(29)}$. Middle-aged males have been shown to have a higher risk of incident T2D compared with middle-aged females ${ }^{(25,30,31)}$. Therefore, if indeed low vitamin D levels contribute to the pathogenesis of T2D, our analysis of 32-year-olds might be expected to show a 
stronger relationship in males than females, who are not expected to develop disease until later.

A prospective cohort study among non-diabetic participants found that lower concentration of baseline 25(OH)D is associated with higher fasting insulin and HOMA-IR, along with other indicators of impaired glucose metabo$\operatorname{lism}^{(32)}$. However, they were unable to study incident T2D due to a small number of cases. Conversely, a study by Mattila et al. reported that the association of $25(\mathrm{OH}) \mathrm{D}$ concentration with incident T2D was attenuated by the addition of concurrent BMI into the model, although the trend of increasing T2D diagnosis with decreasing quartile of serum $25(\mathrm{OH}) \mathrm{D}$ remained ${ }^{(33)}$. Unfortunately, they did not examine markers of insulin resistance and so these two studies are not directly comparable with ours. A randomized controlled trial of vitamin D supplementation found that supplemented, obese, non-diabetic AfricanAmerican adolescents had improved fasting insulin and HOMA-IR compared with placebo after 6 months ${ }^{(34)}$, indicating that statistically significant improvements may take some time to be observable even in high-risk populations.

At the population level, most cross-sectional studies of $25(\mathrm{OH}) \mathrm{D}$ and insulin sensitivity have found associations between the two ${ }^{(35)}$. However, other cross-sectional studies have found that insulin sensitivity is independent of $25(\mathrm{OH}) \mathrm{D}$ in specific populations, such as African Americans ${ }^{(36,37)}$ and the morbidly obese ${ }^{(38)}$. Beyond the associations between vitamin D and adiposity, evidence from basic science research points to mechanisms for potential associations between vitamin $\mathrm{D}$ and glucose homeostasis at the cellular level. Vitamin D receptors ${ }^{(39)}$ and $1 \alpha$-hydroxylase ${ }^{(40)}$ are expressed on insulin-producing pancreatic $\beta$ cells. The promoter of the human insulin gene has a vitamin D response element ${ }^{(41)}$, while 1,25-dihydroxyvitamin D activates transcription of that gene ${ }^{(42)}$. Evidence has also been found in animal studies. A study in mice found that lack of a functional vitamin $\mathrm{D}$ response impaired insulin response $^{(43)}$. In an in vivo rat study, vitamin D deficiency impaired glucose-mediated insulin secretion, which was restored by supplementation ${ }^{(44)}$.

Intervention studies of vitamin D supplementation have not found an association between supplementation and hepatic insulin sensitivity nor with direct measures of insulin sensitivity ${ }^{(35)}$. Some of these inconsistencies may be related to sex-specific or population-level differences in the relationships.

The major strength of the present study is the data on lifestyle characteristics, demographics and CMR in young adult offspring. That our analyses specifically examine sex-specific differences as well as the role of obesity in these relationships are additional strengths. By examining these relationships in a young, healthy population, we have fewer confounding factors and co-morbidities to consider. However, limitations of our study deserve mention. Levels of 25(OH)D and CMR were measured cross-sectionally and so the temporal relationship of observed associations remains unclear. We tested associations with eleven different CMR outcomes, leading to a substantial correction for multiple testing. Finally, specific characteristics of the study population (e.g. lifestyle and dietary characteristics) may limit generalizability of our findings. Similarities of our findings, particularly overall findings, with previous reports mitigate this concern.

\section{Conclusion}

In conclusion, we report that an inverse association of $25(\mathrm{OH}) \mathrm{D}$ with TAG is attenuated after adjustment by current BMI in young adults. We also report that $25(\mathrm{OH}) \mathrm{D}$ is associated with insulin sensitivity markers in young adult males, but not females. Inconsistencies that are observed, particularly in relation to sex-specific differences and the absence of similar associations in intervention studies, indicate that the relationship between vitamin $\mathrm{D}$ and insulin sensitivity may be more complex than anticipated. It would be interesting to conduct follow-up studies in this and similar populations as the participants age. Better understanding of these relationships may help direct future research for public health or clinical applications. Finally, well-designed randomized controlled trials of vitamin D supplementation and CMR are needed in well-defined at-risk populations to determine the utility of vitamin D supplementation in preventing cardiometabolic disease.

\section{Acknowledgements}

Financial support: This work was supported by grants R01HL088884 (Principal Investigator: D.S.S.) and K01HL103174 (Principal Investigator: D.A.E.) from the National Heart, Lung, and Blood Institute of the National Institutes of Health (NIH). The work was partially supported by the Nutrition and Obesity Research Center at the University of Washington (grant number P30 DK035816). A.M. received support from the National Institutes of Child Health and Development of the NIH (grant number T32 HD052462). The funding bodies had no role in the design, analysis or writing of this article. Conflicts of interest: None. Authorship: A.M. designed the research, analysed the data, wrote the paper and had primary responsibility for the final content. H.H. conducted the research and contributed to the paper. C.M.S. analysed the data and contributed to the paper. M.A.W. contributed to the paper. A.N.H. contributed to the paper. I.H.d.B. contributed to the paper. B.K. contributed to the paper. D.S.S. designed the research and contributed to the paper. Y.F. designed the research and contributed to the paper. D.A.E. designed the research and contributed to the paper. All authors read and approved the final manuscript. Ethics of human subject participation: Study procedures were approved by the Institutional Review Boards of the University of Washington, 
in Seattle, and the Hebrew University Hadassah Medical Center in Jerusalem. All participants provided informed consent.

\section{References}

1. Holick MF (2004) Sunlight and vitamin D for bone health and prevention of autoimmune diseases, cancers, and cardiovascular disease. Am J Clin Nutr 80, 6 Suppl., 1678S-16788S

2. Cranney A, Weiler H, O'Donnell S et al. (2008) Summary of evidence-based review on vitamin D efficacy and safety in relation to bone health. Am J Clin Nutr 88, issue 2, 513S-519S.

3. Holick MF (2007) Vitamin D deficiency. N Engl J Med 357, 266-281.

4. Giovannucci E, Liu Y, Hollis B et al. (2008) 25Hydroxyvitamin D and risk of myocardial infarction in men: a prospective study. Arch Intern Med 168, 1174-1180.

5. Zittermann A, Gummert JF \& Borgermann J (2009) Vitamin D deficiency and mortality. Curr Opin Clin Nutr Metab Care 12, 634-639.

6. Wang TJ, Pencina MJ, Booth SL et al. (2008) Vitamin D deficiency and risk of cardiovascular disease. Circulation 117, 503-511.

7. Judd SE \& Tangpricha V (2009) Vitamin D deficiency and risk for cardiovascular disease. Am J Med Sci 338, 40-44.

8. Fiscella K \& Franks P (2010) Vitamin D, race, and cardiovascular mortality: findings from a national US sample. Ann Fam Med 8, 11-18.

9. van Lennep JER, Westerveld HT, Erkelens DW et al. (2002) Risk factors for coronary heart disease: implications of gender. Cardiovasc Res 53, 538-549.

10. Hochner H, Friedlander Y, Calderon-Margalit R et al. (2012) Associations of maternal prepregnancy body mass index and gestational weight gain with adult offspring cardiometabolic risk factors: the Jerusalem Perinatal Family Follow-up Study. Circulation 125, 1381-1389.

11. Holick MF, Binkley NC, Bischoff-Ferrari HA et al. (2011) Evaluation, treatment, and prevention of vitamin D deficiency: an Endocrine Society clinical practice guideline. $J$ Clin Endocrinol Metab 96, 1911-1930.

12. Jorde R \& Grimnes G (2011) Vitamin D and metabolic health with special reference to the effect of vitamin $D$ on serum lipids. Prog Lipid Res 50, 303-312.

13. Kayaniyil S, Vieth R, Harris SB et al. (2011) Association of 25 (OH)D and PTH with metabolic syndrome and its traditional and nontraditional components. J Clin Endocrinol Metab 96, $168-175$.

14. Zittermann A, Frisch S, Berthold HK et al. (2009) Vitamin D supplementation enhances the beneficial effects of weight loss on cardiovascular disease risk markers. Am J Clin Nutr 89, $1321-1327$.

15. Jorde R, Sneve M, Torjesen P et al. (2010) No improvement in cardiovascular risk factors in overweight and obese subjects after supplementation with vitamin $\mathrm{D}_{3}$ for 1 year. $J$ Intern Med 267, 462-472.

16. Heikkinen A-M, Tuppurainen MT, Niskanen L et al. (1997) Long-term vitamin $\mathrm{D}_{3}$ supplementation may have adverse effects on serum lipids during postmenopausal hormone replacement therapy. Eur J Endocrinol 137, 495-502.

17. Andersen R, Brot C, Mejborn H et al. (2009) Vitamin D supplementation does not affect serum lipids and lipoproteins in Pakistani immigrants. Eur J Clin Nutr $\mathbf{6 3}$, 1150-1153.

18. Nagpal J, Pande JN \& Bhartia A (2009) A double-blind, randomized, placebo-controlled trial of the short-term effect of vitamin $\mathrm{D}_{3}$ supplementation on insulin sensitivity in apparently healthy, middle-aged, centrally obese men. Diabet Med 26, 19-27.

19. Reid IR, Mason B, Horne A et al. (2002) Effects of calcium supplementation on serum lipid concentrations in normal older women: a randomized controlled trial. Am J Med 112, 343-347.

20. Zemel MB, Shi H, Greer B et al. (2000) Regulation of adiposity by dietary calcium. FASEB J 14, 1132-1138.

21. Kamycheva E, Jorde R, Figenschau Y et al. (2007) Insulin sensitivity in subjects with secondary hyperparathyroidism and the effect of a low serum 25-hydroxyvitamin D level on insulin sensitivity. J Endocrinol Invest 30, 126-132.

22. Yin X, Sun Q, Zhang X et al. (2012) Serum 25(OH)D is inversely associated with metabolic syndrome risk profile among urban middle-aged Chinese population. Nutr J 11, 68.

23. Delvin EE, Lambert M, Levy E et al. (2010) Vitamin D status is modestly associated with glycemia and indicators of lipid metabolism in French-Canadian children and adolescents. J Nutr 140, 987-991.

24. Husemoen LL, Thuesen BH, Fenger M et al. (2012) Serum $25(\mathrm{OH}) \mathrm{D}$ and type 2 diabetes association in a general population: a prospective study. Diabetes Care $\mathbf{3 5}$, $1695-1700$.

25. Choi YJ, Kim HC, Kim HM et al. (2009) Prevalence and management of diabetes in Korean adults: Korea National Health and Nutrition Examination Surveys 1998-2005. Diabetes Care 32, 2016-2020.

26. Fraser A, Williams D \& Lawlor DA (2010) Associations of serum 25-hydroxyvitamin D, parathyroid hormone and calcium with cardiovascular risk factors: analysis of 3 NHANES cycles (2001-2006). PLoS One 5, e13882.

27. Liu E, Meigs JB, Pittas AG et al. (2009) Plasma 25hydroxyvitamin D is associated with markers of the insulin resistant phenotype in nondiabetic adults. $J$ Nutr 139, 329-334.

28. Del Gobbo LC, Song Y, Dannenbaum DA et al. (2011) Serum 25-hydroxyvitamin $D$ is not associated with insulin resistance or $\beta$ cell function in Canadian Cree. J Nutr 141, 290-295.

29. Geer EB \& Shen W (2009) Gender differences in insulin resistance, body composition, and energy balance. Gend Med 6, Suppl. 1, 60-75.

30. Lipscombe LL \& Hux JE (2007) Trends in diabetes prevalence, incidence, and mortality in Ontario, Canada 1995-2005: a population-based study. Lancet 369, 750-756.

31. Collaboration TERF (2010) Diabetes mellitus, fasting blood glucose concentration, and risk of vascular disease: a collaborative meta-analysis of 102 prospective studies. Lancet 375, 2215-2222.

32. Forouhi NG, Luan J, Cooper A et al. (2008) Baseline serum 25-hydroxy vitamin $\mathrm{D}$ is predictive of future glycemic status and insulin resistance: the Medical Research Council Ely Prospective Study 1990-2000. Diabetes 57, 2619-2625.

33. Mattila C, Knekt P, Mannisto S et al. (2007) Serum 25hydroxyvitamin $\mathrm{D}$ concentration and subsequent risk of type 2 diabetes. Diabetes Care 30, 2569-2570.

34. Belenchia AM, Tosh AK, Hillman LS et al. (2013) Correcting vitamin D insufficiency improves insulin sensitivity in obese adolescents: a randomized controlled trial. Am J Clin Nutr 97, 774-781.

35. Pittas AG, Lau J, Hu FB et al. (2007) The role of vitamin D and calcium in type 2 diabetes. A systematic review and meta-analysis. J Clin Endocrinol Metab 92, 2017-2029.

36. Scragg R, Sowers M \& Bell C (2004) Serum 25hydroxyvitamin $\mathrm{D}$, diabetes, and ethnicity in the Third National Health and Nutrition Examination Survey. Diabetes Care 27, 2813-2818.

37. Alemzadeh R, Kichler J, Babar G et al. (2008) Hypovitaminosis D in obese children and adolescents: relationship with 
adiposity, insulin sensitivity, ethnicity, and season. Metabolism 57, 183-191.

38. Manco M, Calvani M, Nanni G et al. (2005) Low 25hydroxyvitamin D does not affect insulin sensitivity in obesity after bariatric surgery. Obes Res 13, 1692-1700.

39. Johnson J, Grande J, Roche P et al. (1994) Immunohistochemical localization of the $1,25(\mathrm{OH})_{2} \mathrm{D}_{3}$ receptor and calbindin D28k in human and rat pancreas. Am J Physiol Endocrinol Metab 267, E356-E360.

40. Bland R, Markovic D, Hills CE et al. (2004) Expression of 25hydroxyvitamin $\mathrm{D}_{3}-1 \alpha$-hydroxylase in pancreatic islets. J Steroid Biochem Mol Biol 89-90, 121-125.
41. Maestro B, Dávila N, Carranza MC et al. (2003) Identification of a vitamin $\mathrm{D}$ response element in the human insulin receptor gene promoter. J Steroid Biochem Mol Biol 84, 223-230.

42. Maestro B, Molero S, Bajo S et al. (2002) Transcriptional activation of the human insulin receptor gene by $1,25-$ dihydroxyvitamin $\mathrm{D}_{3}$. Cell Biochem Funct 20, 227-232.

43. Zeitz U, Weber K, Soegiarto D et al. (2003) Impaired insulin secretory capacity in mice lacking a functional vitamin D receptor. FASEB J 17, 509-511.

44. Cade C \& Norman AW (1986) Vitamin $\mathrm{D}_{3}$ improves impaired glucose tolerance and insulin secretion in the vitamin D-deficient rat in vivo. Endocrinology 119, 84-90. 